\title{
Relationship between Stage of Change for Three Health Behaviors and Perceived Stress in Chinese Adults
}

\author{
Ke Deng1, Akira Tsuda ${ }^{2}$ \\ ${ }^{1}$ Institute of Comparative Studies of International Cultures and Societies, Kurume University, Kurume, Japan \\ ${ }^{2}$ Department of Psychology, Kurume University, Kurume, Japan \\ Email: sztouka@yahoo.co.jp
}

Received 14 September 2015; accepted 26 September 2015; published 30 September 2015

\begin{abstract}
We propose stress management behavior, exercise, and scheduled sleep to represent the general intention and actual practices aimed to relieve stress by the application of the transtheoretical model (TTM). Randomly selected Chinese adults aged 18 - 64 were tested through an internet survey. The stage distributions for the three health behaviors were different across the Chinese adults. Stress was significantly higher in action and maintenance stages than in precontemplation and contemplation stages across three health behaviors. Our findings have provided preliminary findings on the applicability of TTM on the three health behaviors in Chinese adults with evidence of concurrent criterion validity.
\end{abstract}

\section{Keywords}

Stage of Change, Exercise, Sleep Behavior, Stress Management

\section{Introduction}

Public health programs that are aimed at combating stress will have to include health behavior change as a major focus. Stressful experiences have been found to be linked to numerous chronic diseases such as cardiovascular diseases [1]. Thus, it is important to prevent stress-related health problems [2]. Approaches for assessing stress focus on individual health behavior. For example, stress was reported to be simultaneously associated with physical inactivity [3], unhealthy sleep behavior [4] and stress management behavior [5]. This interrelationship between specific behaviors contributes to a greater or lesser extent to the practice of specific health-related behaviors. Here, we assume that people with the general intention to relieve stress are more likely to adopt specific health promotion activities. In contrast, stressed people who are unaware of health promotion are unwilling to proactively engage in desirable health-related behaviors such as regular physical and healthy sleep. We further assume that this general intention and actual practice to relieve stress is associated with health behavior that may be intended for relieving stress. Moreover, although there are considerable overlaps in the constructs which major health behavior models identify as important in predicting behavior [6], intention plays a role in many of the most widely applied models [7].

How to cite this paper: Deng, K. and Tsuda, T. (2015) Relationship between Stage of Change for Three Health Behaviors and Perceived Stress in Chinese Adults. Open Journal of Social Sciences, 3, 295-299. 
Based on this concept, we first assess whether an individual has any intention to adopt any practice to promote health. If so, we find out if there is an actual plan to execute the practice and which stage it has reached. The transtheoretical model (TTM) is used to describe people intending to change behaviors through five stages, i.e., precontemplation (there is no intention to change in the next six months), contemplation (intending to change in the next six months), preparation (intending to change in the next one month), action (practice to change lasts less than six months), and maintenance (practice to change lasts at least six months) [8]. Although the TTM has been applied widely to health behaviors in western countries, few studies have been conducted in Asian populations, not mention the specific behaviors of exercise, stress management, and sleep behavior. This study had three principal goals: 1) to collect information from participants in terms of the five TTM stages as basis for future comparison and intervention; 2) to correlate the information gathered with three health behaviors; and 3) to study whether different stages of change in health behaviors are positively related to stress-relief.

\section{Method}

\subsection{Study Design and Sample}

This study was a longitudinal study which was conducted three times. This study provides the results of the first leg. The participants were Chinese, aged 18 - 64 years old who were randomly selected using multistage sampling. A total of 1599 respondents were successfully interviewed from March to September 2014.

\subsection{Instrument}

In this study, participants were eventually classified into five stages: 1) "No. I have no intention to begin in the next sixmonths.” (precontemplation); 2) “No. But I intend to begin in the next six months.” (contemplation); 3) "No. But I intend to begin in the next month." (preparation); 4) "Yes. I have been practicing for less than sixmonths.” (action); or 5) "Yes. I have been practicing for at least six months.” (maintenance).

Health behaviors were measured regardless of whether they were intended to promote health. Exercise was assessed by asking the participants whether they had performed exercises that lasted for at least 30 min in the past 1 month [9]. Effective stress management was referred to any form of healthy activity practiced at least 20 min per day to manage stress [5]. Scheduled sleep was defined by asking the participants whether they woke up at the same time everyday (with an acceptable difference of $30 \mathrm{~min}$ ). To examine the validity of the stage of scheduled sleep, the relationships between the stages and stress were examined using ANOVAs. Sample characteristics assessed included gender, age, education, marital status, and perceived stress. Stress was assessed by means of the Chinese Perceived Stress Scale.

\subsection{Statistical Analysis}

All analyses were conducted using SPSS for Windows version 22.0. Descriptive statistics were used to describe sample characteristics and stage distribution for the three selected health behaviors. Three one-way analyses of variance (ANOVAs) were conducted. The model assumes that stress causes stage transitions [10]; thus, they were entered into the ANOVAs as independent variables. However, this study aimed to examine the relationship between the stages and stress. In previous cross-sectional studies of the relationships between depression and stages of change for effective stress management, depression was entered into the analyses as a dependent variable [10]. Therefore, this study described stress as the dependent variable.

\section{Results}

\subsection{Sample Characteristics}

Table 1 indicates that more half of the participants were female. Age was normally distributed, with the age group 25 - 34 years old being the largest category. Two-thirds of the participants were married. Most of the participants were college education. The demographic characteristics of this sample were comparable to those of the general Chinese population.

\subsection{Stage Distribution}

Table 2 provides the estimate of stage distribution of the three health behaviors. The pattern of similarities and 
Table 1. Sample characteristics.

\begin{tabular}{|c|c|c|c|}
\hline Variable & Leveles & $\mathrm{n}$ & $\%$ \\
\hline \multirow[t]{2}{*}{ Gender } & Feamale & 824 & $51.5 \%$ \\
\hline & Male & 775 & $48.5 \%$ \\
\hline \multirow[t]{5}{*}{ Age } & $18-24$ & 299 & $18.7 \%$ \\
\hline & $25-34$ & 854 & $53.4 \%$ \\
\hline & $35-44$ & 345 & $21.6 \%$ \\
\hline & $45-54$ & 88 & $5.5 \%$ \\
\hline & $55-64$ & 13 & $0.8 \%$ \\
\hline \multirow{2}{*}{ Marital statı } & Married & 1040 & $65.0 \%$ \\
\hline & Single & 559 & $35.0 \%$ \\
\hline \multirow[t]{4}{*}{ Education } & $<$ High scho & 4 & $0.3 \%$ \\
\hline & High schoo & 64 & $4.0 \%$ \\
\hline & Some colle & 1369 & $85.6 \%$ \\
\hline & Grad/post & 162 & $10.1 \%$ \\
\hline
\end{tabular}

Table 2. Distribution across stages of change for the three health behaviors.

\begin{tabular}{|l|c|c|c|c|c|l|}
\hline & PC & C & PR & A & M & \\
\hline Stress management behavior & 162 & 305 & 285 & 430 & 313 & $P<0.01$ \\
\hline & $10.8 \%$ & $20.4 \%$ & $19.1 \%$ & $28.8 \%$ & $20.9 \%$ & \\
\hline Exercise & 87 & 193 & 529 & 273 & 358 & $P<0.01$ \\
\hline Scheduled sleep & $6 \%$ & $13.4 \%$ & $36.7 \%$ & $19 \%$ & $24.9 \%$ & \\
\hline & 155 & 226 & 473 & 293 & 452 & $P<0.01$ \\
\hline
\end{tabular}

differences in stage distribution is more readily viewed graphically, and each health behavior is discussed below in conjunction with Figure 1.

\subsection{Relationships between Stages and Stress}

The ANOVA was conducted with Tukey's post-hoc tests with stage as the independent variable. It revealed significant effects of each stage (Stress management: $F(4,1490)=30.78, \mathrm{p}<0.01, \eta^{2}=0.08$; Exercise: $\mathrm{F}(4,1435)=$ 36.98, $\mathrm{p}<0.01, \eta^{2}=0.09$; Scheduled sleep: $\left.\mathrm{F}(4,1594)=56.90, \mathrm{p}<0.01, \eta^{2}=0.14\right)$. The results of post-hoc comparisons indicated that the scores for stress were significantly higher in action and maintenance than in precontemplation and contemplation for the three health behaviors (all, $\mathrm{p}<0.01$ ) (Figure 1).

\section{Discussion}

The first objective of this study was to measure the stage of change for exercise, stress management, and sleep behavior in Chinese adults. This study is the first to report data from a sample of Chinese adults on the stages of distribution for the general intention and introduce actual practices to promote health. We have reported unique findings that the stage of change construct of the TTM could measure and distinguish people's readiness to take action to promote health.

The "actors" represented the largest group among the five stages of change in stress management behavior, which was largely consistent with the findings of previous studies [7]. The "preparers" represented the largest group among the five stages of change in regulating exercise and scheduling sleep, suggesting that a large part of the adult population was ready to relieve stress by regulated exercise or scheduled sleep. The distinction between "contemplators" and "preparers" is important in relieving stress. It is because individuals who have not changed yet but are planning to do so in the near future have been grouped with the "precontemplators," who have no intention to change in terms of the traditional dichotomous categorization of health behaviors. Finally, the "actors" and "maintainers" represent the more health-conscious portion of the population. The "maintainers," who have been engaging in health promotion action for a substantial period, represent a desirable target market for health promotion programs. However, we cannot rule out the possibility of inflated figures in the number of actors and maintainers due to over-reporting of socially-desirable behavior. 


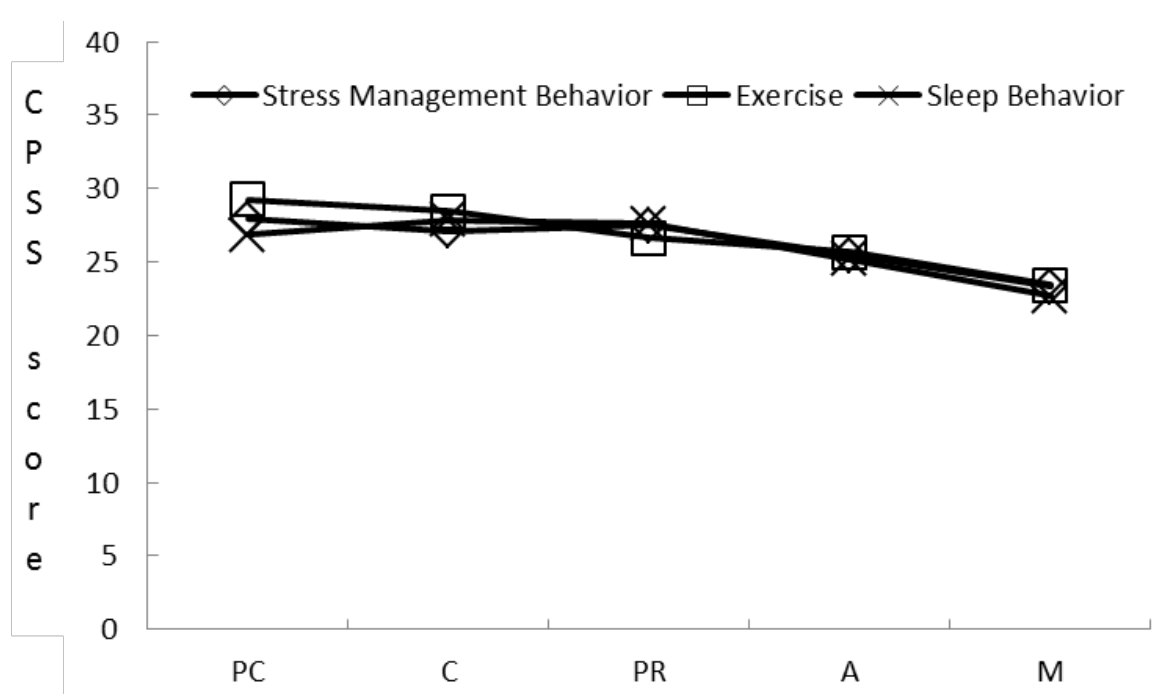

Figure 1. Differences in the scores for the perceived stress across the stages of change for three health behaviors. Note: $\mathrm{PC}=$ precontemplation; $\mathrm{C}=$ contemplation; $\mathrm{PR}=$ preparation; $\mathrm{A}=$ action; $\mathrm{M}=$ maintenance.

The second objective of this study was to determine whether stages of change for the three health behaviors were positively associated with stress. This was an attempt to examine the concurrent criterion validity of the stages of change for the three health behaviors. Our results indicated that the stages of change for the three health behaviors were positively associated with stress levels.

Previous studies on stage for various health behaviors [2] [10] found that participants in later stages were more likely to report higher involvement in the respective specific behavior than participants in earlier stages. Our results therefore extend these studies by showing that such relationships can also be observed between the stages for three health behaviors.

The results also provide strong support for the applicability of the TTM among Chinese adults. To our knowledge, there are few TTM studies in which the samples are Chinese adults. One study is about health behaviors among Chinese in Hong Kong [11], and another is about cessation of exercise among Chinese university students [9]. One of the weaknesses of these TTM studies was that most of them relied on single stage samples from specific settings that might limit generalizability of their findings. Our findings have the strength of being based on a representative sample of the general population.

This study has several limitations. First, our findings were based on cross-sectional analyses; thus, we could not determine which the cause was and which the effect was. Movement between stages in health behaviors over time could not be assessed here. To conclude, our findings have provided unique preliminary findings on the application of TTM's stages of change with regard to the general intention and actual practices aimed at promoting health in a representative sample of the Chinese adult population with evidence of concurrent criterion validity. Although further research, especially using prospective design, is needed to confirm our findings, we suggest that periodic surveillance of the general intention to promote health in the general population would provide useful information to health planners about the population's receptiveness for health promotion interventions aimed at health behavior changes.

\section{References}

[1] McEwen, B.S. (2007) Physiology and Neurobiology of Stress and Adaptation: Central Role of the Brain. Physiological Reviews, 87, 873-904. http://dx.doi.org/10.1152/physrev.00041.2006

[2] Horiuchi, S., Tsuda, A., Prochaska, J.M., Kobayashi, H. and Mihara, K. (2012) Relationships between Stages and Processes of Change for Effective Stress Management in Japanese College Students. Psychology, 3, 494-499. http://dx.doi.org/10.4236/psych.2012.36070

[3] Rebar, A.L., Duncan, M.J., Short, C. and Vandelanote, C. (2014) Differences in Health-Related Quality of Life between Three clusters of Physical Activity, Sitting Time, Depression, Anxiety, and Stress. BMC public Health, 14, 1088. http://dx.doi.org/10.1186/1471-2458-14-1088 
[4] Wolkow, A., Ferguson, S., Aisbett B., and Main L. (2015) Effects of Work-Related Sleep Restriction on Acute Physiological and Psychological Stress Responses and Their Interactions: A Review among Emergency Service Personnel. International Journal of Occupational Medicine and Environmental Health, 28.

[5] Deng, K., Tsuda, A., Horiuchi, S. and Matsuda, T. (2013) Relationships between Stages and Processes of Change for Effective Stress Management in Chinese College Students. Stress Science Research, 28, 74-81. http://dx.doi.org/10.5058/stresskagakukenkyu.28.74

[6] Conner, M and Norman, P. (1996) The Role of Social Cognition in Health Behaviors. In: Conner, M. and Norman, P., Eds., Predicting Health Behavior, Open University Press, Buckingham, 1-21.

[7] Ajzen, I. (1991) The Theory of Planned Behavior. Organizational Behavior and Human Decision Processes, 50, 179211. http://dx.doi.org/10.1016/0749-5978(91)90020-T

[8] Prochaska, J.O. and DiClemente, C.C. (1983) Stages and Processes of Self-Change of Smoking: Toward an Integrative Model of Change. Journal of Consulting and Clinical Psychology, 51, 390-395. http://dx.doi.org/10.1037/0022-006X.51.3.390

[9] Si, Q. (2005) Study on Phase Change and Mental Decisive Factors of Sport Exercise Behavior of College Students. China Sport Science, 25, 76-83.

[10] Kim, E., Tsuda, A., Horiuchi, S., Park, Y.-S., Kim, U. and Hong, K.-S. (2009) The Relationship between Stages of Change for Stress Management and Depressive Symptoms in a Sample of Korean University Students. Kurume University Psychological Research, 8, 103-110.

[11] Lam, T.H., Chan, B., Ho, S.Y. and Chan, W.M. (2004) Stage of Change for General Health Promotion Action and Health-Re- lated Lifestyle Practices in Chinese Adults. Preventive Medicine, 38, 302-308. http://dx.doi.org/10.1016/j.ypmed.2003.10.005 